\title{
Spectra of Hot Molecules of Astrophysical Importance: an Update on the ExoMol Project
}

\author{
J. Tennyson and S. N. Yurchenko
}

Department of Physics and Astronomy, University College London, London WC1E 6BT, UK

\begin{abstract}
The ExoMol project aims at providing comprehensive line lists of molecular transitions which are likely to be important in the atmospheres of extra-solar planets and cool stars, including brown dwarfs. The physics of the molecules involved varies and can be categories according to the number atoms in the species. Thus the molecules can be grouped as diatomics, both closed and open and shell, triatomics, tetratomics, methane and larger species. Progress in providing complete sets of line list for the molecules in each of these categorizes is considered. The latest line lists and related data for each of the species can be found on the project website www. exomol.com.
\end{abstract}

Keywords. molecular data; opacity; astronomical data bases: miscellaneous; planets and satellites: atmospheres; stars: low-mass

\section{Introduction}

Most of the Universe is molecular and most of the Universe is also very cold. However there are a significant number of places where processes involving the spectra of hot molecules are highly important. One such situation occurs when molecules are strongly out of local thermodynamic equilibrium. Examples include masers and the emission spectra observed from cometary atmospheres following solar pumping (Dello Russo et al. 2005), a situation which is likely to occur in other near-stellar regimes. Alternatively molecules can exist in hot environments such as those found in the atmospheres of cool stars and brown dwarfs (Allard et al. 1997), and even in sunspots (Polyansky et al. 1997b). More recently the beginnings of the process of identifying of molecules in the atmospheres of exoplanets (Tinetti et al. 2007; Swain et al. 2008) has led to a new and active field in the spectroscopy of hot molecules. Of course, one cannot expect all exoplanets to have hot atmospheres, but so far these are ones which are amenable to observation.

The spectroscopy of most molecules becomes complicated at elevated temperatures because of the sheer number of energy levels that can be thermally occupied. As will be discussed below, this can lead to the spectrum of a single molecule requiring billions of transitions to properly represent it. This clearly puts huge demands on laboratory astrophysics to provide the appropriate data. The ExoMol project (Tennyson \& Yurchenko 2012) has been created to meet precisely these demands. Although the direct scope of the project is the exoplanet spectroscopy, it has many other applications to both astrophysical and non-astrophysical problems. Here we summarise the current progress in providing the molecular line lists required by this project. 


\section{Methodology}

The methodology used by ExoMol (see Fig. 1), and indeed by a number of other similar, more focused studies, has been well documented elsewhere (Tennyson 2012; Tennyson \& Yurchenko 2012), so only an overview will be provided here.

The ExoMol project relies very heavily on the application of ab initio quantum mechanics. Operating within the standard Born-Oppenheimer approximation, this is done in two steps. First, detailed and extensive electronic structure calculations are performed to characterise the potential energy surface (PES), or for diatomics the potential energy curves. At the same time the dipole moment surface (DMS) for the molecule is also computed. In the second step, the nuclear motion problem is solved for the given PES. For diatomics this can be done by direct solution of the Schrödinger equation, while for polyatomic molecules procedures based on the variational principle (Tennyson et al. 2004; Kozin et al. 2004; Schwenke 1996; Yurchenko et al. 2007) are used. The wave functions from this step are then used with the DMS to calculate transition intensities, usually by computing the Einstein-A coefficient for each transition. Finally we note that although here and below we use the language of the Born-Oppenheimer approximation, one has to relax it for calculations which approach spectroscopic accuracy (Zobov et al. 1996) and many of the studies discussed below do indeed make allowance for effects beyond the Born-Oppenheimer approximation. Lodi \& Tennyson (2010) give a tutorial on how to calculate spectra from first principles using water as an example.

Our ability to compute molecular spectra using a purely ab initio approach is improving rapidly (Boyarkin et al. 2013; Polyansky et al. 2013b,a). However obtaining line lists with spectroscopic accuracy by purely ab initio means is thus far confined to systems with very few electrons such as $\mathrm{H}_{3}^{+}$(Neale et al. 1996; Polyansky \& Tennyson 1999; Pavanello et al. 2012), $\mathrm{HeH}^{+}$(Engel et al. 2005) and other species found in the primordial Universe (Coppola et al. 2011). Otherwise it is necessary to use experimental data to obtain wavelengths of the accuracy required for applications. This can be done using three distinct techniques. Most commonly the computed PES is adjusted to better reproduce the laboratory data; techniques for doing this are well advanced (Yurchenko et al. 2011b). The second technique notes that in ab initio vibration-rotation spectra, the dominant error arises in the vibrational band origins (Polyansky et al. 1997a). Nuclear motion programs, such as TROVE (Yurchenko et al. 2007), which use a pure vibrational wave function as a basis for solving the full rotation-vibration problem, admit the possibility of adjusting the vibrational band origins to the empirical values before addressing the rotational problem (see Yurchenko et al. 2009; Sousa-Silva et al. 2013). If necessary, this can be combined with judicious adjustment of the equilibrium geometry in the PES (Sousa-Silva et al. 2013), to give a good rotational spectrum. Finally, the calculated energy levels can be replaced by experimental ones, if available, as has been done by Harris et al. (2006) and Lodi \& Tennyson (2012). In practice more than one of these approaches can, and often is, used in a single calculation.

The situation with the DMS is very different. Experience has shown that this is best computed ab initio (Lynas-Gray et al. 1995). Furthermore techniques are being developed which allows the DMS to computed to high accuracy (Lodi et al. 2011), arguably giving transition intensities more accurately than they can routinely be measured.

\section{Current status}

We recently summarised the current status of line lists for hot molecules from all sources (Tennyson \& Yurchenko 2012), so here we will concentrate on the progress that 


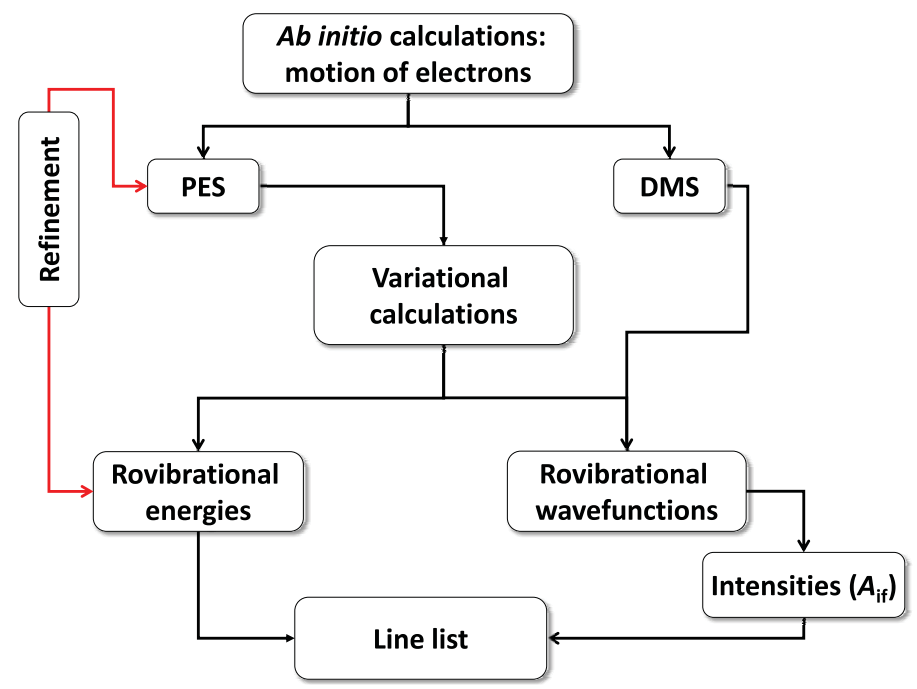

Figure 1. Schematic of the general method used to produce molecular line lists.

has been made in the ExoMol project in supplying reliable data in the many cases where this is not available. In doing this we will consider the molecules in order of complexity which is approximately given by the number of degrees of freedom that have to be considered in the nuclear motion problem.

\subsection{Closed shell diatomics}

The following experimental diatomic line lists are already available and should be sufficient for accurate modelling hot atmospheric environments: HITEMP (Rothman et al. 2010) contains extensive line lists for CO and NO; a comprehensive hot line list for the weak, quadruple-allowed transitions of molecular hydrogen has been constructed recently by Campargue et al. (2012); a line list for $\mathrm{O}_{2}$ is available from $\mathrm{Yu}$ et al. (2012).

Closed shell diatomic molecules are straightforward to deal with. Provided there is a significant amount of experimental data, and for the species under consideration by ExoMol there generally is, then this can be used to effectively characterise the ground state potential energy curve. Although it is usual to start refinement from an ab initio curve, experience has shown that using Leroy's DPotFit potential curve fitting program (Le Roy 2006) along with vibration-rotation transitions or energy levels is sufficient to characterise the curve. The experimental dissociation energy, $D_{0}$, if available, can be used to constrain the asymptotic behaviour of the curve which helps greatly in giving reliable estimates for the positions of high-lying vibrational states for which no measured data is available.

Final calculations can be performed program LEVEL due to Le Roy (2007). Of course this also involves the calculation of transition intensities, or more accurately Einstein A coefficients. For this (empirical) potential energy curve must be complemented by a curve giving the value of the dipole moment as a function of internuclear separation. Although such curves have been determined empirically ( $\mathrm{Li}$ et al. 2013), our preferred option is to use high quality $a b$ initio calculations for this. Such calculations are not only proving highly accurate, they also allow the dipole curve to be evaluated for geometries not yet probed by experimental spectra. This is important for characterising spectra involving 


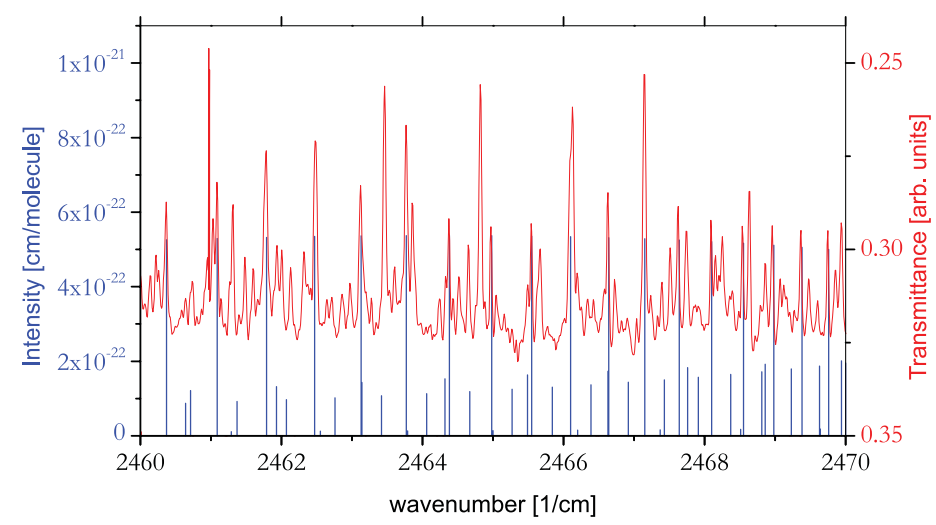

Figure 2. Sunspot absorption spectra (Wallace et al. 1996), continuous line and predicted ${ }^{28} \mathrm{Si}^{16} \mathrm{O}$ absorptions at $3200 \mathrm{~K}$ due to Barton et al. (2013), vertical lines. The absolute intensity scale refers to the calculated spectra.

high-lying states. Such states are particularly sensitive to elongated bondlengths which cause their own issues as coupled cluster methods such as the "gold standard" CCSD(T) can behave badly near dissociation resulting in unphysical dipole moment curves (see Barton et al. 2013, for example). Our preference is therefore to employ multi-reference methods such as MRCI for this step of the calculation.

A recently completed example of this approach was the study of $\mathrm{SiO}$ by Barton et al. (2013). Here accurate rotation-vibration line lists were calculated for all the main isotopologues of silicon monoxide: ${ }^{28} \mathrm{Si}^{16} \mathrm{O},{ }^{29} \mathrm{Si}^{16} \mathrm{O},{ }^{30} \mathrm{Si}^{16} \mathrm{O},{ }^{28} \mathrm{Si}^{18} \mathrm{O}$ and ${ }^{28} \mathrm{Si}^{17} \mathrm{O}$, in their ground electronic states. These line lists considered all bound rotation-vibration states of each system and each contain nearly 2 million lines. They are suitable for temperatures up to $9000 \mathrm{~K}$. As illustrated by Fig. 2, these line lists reproduce the very rich absorption spectra of $\mathrm{SiO}$ recorded at high resolution in sunspots.

A number of similar projects are underway computing line lists for closed shell diatomic molecules including $\mathrm{NaCl}, \mathrm{KCl}$ and $\mathrm{CS}$.

\subsection{Open shell diatomics}

In general, open shell diatomic systems present an altogether different challenge. However, in some cases the spectroscopic transitions of interest are determined by a single, very well separated ground state potential energy curve. Under these circumstances the procedure described above for closed shell diatomics can be adopted with only the additional allowance of coupling between electronic spin and/or orbital angular momentum with rotational angular momentum. Line lists for rotation-vibration transitions with the electronic ground state for $\mathrm{BeH}, \mathrm{MgH}$ and $\mathrm{CaH}$ have recently been computed in this manner by the ExoMol project (Yadin et al. 2012), see Fig. 3.

For the majority of open shell diatomics it is not only necessary to explicitly consider several curves but also the couplings between them. This complicates the problem considerably as it is not practical to characterise both the curves and the couplings using experimental data. This means that it is necessary to determine at least part of the problem, for example the couplings, accurately ab initio. This is often far from straightforward, particularly as many of the key open shell systems involve transition metals whose open d shell electrons are sensitive to relativistic effects and the number of electrons in general make them hard to treat with an accuracy approaching that achieved for 


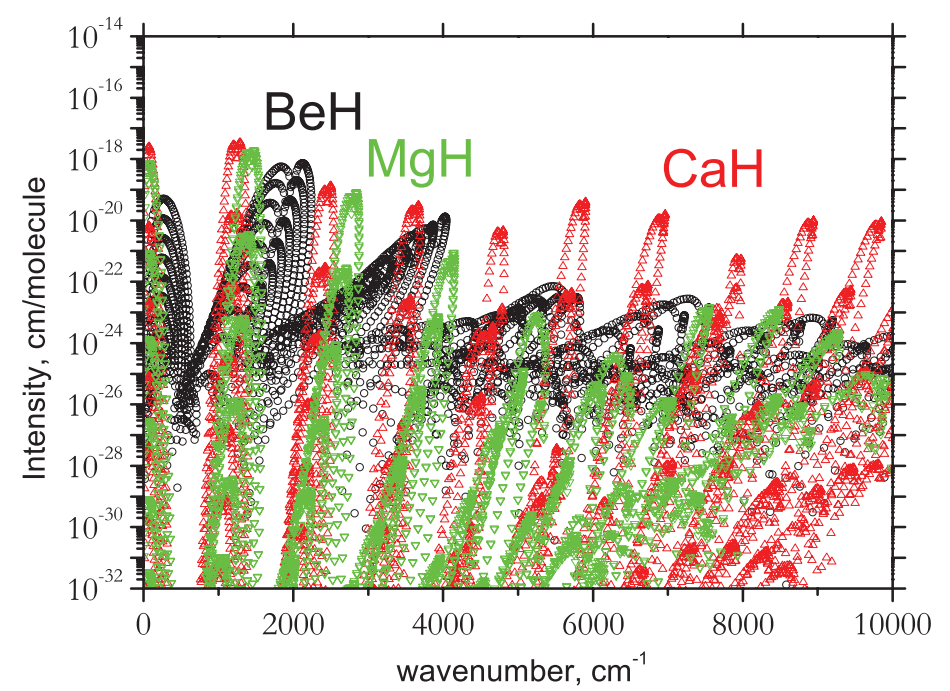

Figure 3. ExoMol absorption spectra of $\mathrm{BeH}, \mathrm{MgH}$, and $\mathrm{CaH}$ at $T=296 \mathrm{~K}$.

more standard, close-shell systems. The TiO line list by Schwenke (1998) was generated by combining experimental data with very demanding electronic structure calculations performed by Langhoff (1997).

The ExoMol project is studying a number of these molecules. Calculations on $\mathrm{AlH}$ (Leyland et al. 2013) and AlO (Patrascu et al. 2013) are nearing completion. At the same time it has been necessary to develop a general coupled-states program for treating the vibration-rotation states of open shell diatomics with a number of nearby electronic states. This program, known as duo, is designed to allow for the inclusion of various coupling terms (spin-orbit, spin-spin, orbit-orbit) in a completely flexible manner.

\subsection{Triatomic species}

The list of triatomics requiring the generation of new vibration-rotation line lists is fairly short. The following hot line lists for triatomic molecules are already available: the BT2 line list for water (Barber et al. 2006); the VTT line list (Voronin et al. 2010) for HDO, the HITEMP line list for $\mathrm{CO}_{2}$ recently improved and extended by Tashkun \& Perevalov (2011); the SMPO (Spectroscopy \& Molecular Properties of Ozone) database of $\mathrm{O}_{3}$; a comprehensive and widely used line list for $\mathrm{H}_{3}^{+}$by Neale et al. (1996). The HCN / HNC line lists of Harris et al. $(2002,2006)$ are currently being improved (Barber et al. 2014) using experimentally determined energy levels (Mellau 2011) to replace the ab initio ones. Similarly the $\mathrm{C}_{3}$ line list of Jørgensen et al. (1989) is only of ab initio quality. Following a preliminary study (Tennyson et al. 2007), this line list is currently being completely recomputed.

A new line list for the $\mathrm{H}_{2} \mathrm{~S}$ molecule has just been completed (Azzam et al. 2013b). Observations of this species are important for determining the sulphur chemistry in exoplanets. However, from a theoretical point of view the spectrum of $\mathrm{H}_{2} \mathrm{~S}$ presents a challenging problem because of the many intensity anomalies caused by having unusually shaped and rather flat DMS (Azzam et al. 2013a). 


\subsection{Tetratomic species}

In contrast to triatomics, there has been little work on complete line lists for tetratomics. So far ammonia is the only species for which comprehensive line list calculations have been completed (Yurchenko et al. 2009, 2011a). Room temperature line lists for $\mathrm{PH}_{3}$ (Sousa-Silva et al. 2013) and $\mathrm{SO}_{3}$ (Underwood et al. 2013) have recently been published. Full, high temperature line lists are currently being computed.

Work has started on $\mathrm{HOOH}$, for which a particularly good ab initio model has been constructed (Polyansky et al. 2013a), and $\mathrm{H}_{2}$ CO. Accurate, hot line lists for such molecules are possible but remain computationally expensive, particularly if they contain more than one heavy atom meaning that they have a low rotational constant. Special computational procedures are being developed to deal with this problem.

An experimental line list for acetylene has been recently constructed, see Moudens et al. (2011), and a line list based on this work is promised. Following initial theoretical studies (Urru et al. 2010), a full line list is also planned as part of the ExoMol project.

\subsection{Methane}

Obtaining a hot line list for methane is of particular importance for the study of exoplanets (Swain et al. 2008; Beaulieu et al. 2011; Waldmann et al. 2013) and other astronomical objects (Geballe et al. 1996; Dinelli et al. 1997). As a consequence a number of groups have reported results aimed at producing a hot line list for this species (Schwenke 2002; Warmbier et al. 2009; Nikitin et al. 2011, 2013; Rey et al. 2013; Wang \& Carrington 2013). Our own work has involved constructing reliable potential energy and dipole moment surfaces (Yurchenko et al. 2013) and producing a preliminary line list (Waldmann et al. 2013). A hot line list containing nearly 10 billion transitions, computed with a specially adapted version of the rotation-vibration program TROVE, is currently being analysed and will be made available in the near future.

\subsection{Larger molecules}

Computing a line list for hot methane is a major computational undertaking. Therefore considering molecules which either contain more atoms or more heavy atoms than methane, or both, is probably not currently possible at the level of accuracy achieved for the species discussed above. Therefore it is necessary to use more approximate methods for solving the rotation-vibration problem. Possible methods include MULTIMODE (Carter \& Bowman 1998) and procedures due to Pavlyuchko et al. (2012). Indeed recently a line list for $\mathrm{C}_{2} \mathrm{H}_{4}$ has been computed using MULTIMODE by Carter et al. (2012), although this line list runs over a limited range of rotational states and has not been made publicly available. Calculations on $\mathrm{C}_{2} \mathrm{H}_{4}$ and $\mathrm{HNO}_{3}$ are currently underway.

\section{Data handling}

Line lists of molecular transitions are often very extensive with a single list for a polyatomic species containing hundreds of millions or even billions of lines. This causes data issues with both storage and use. The format adopted for the ExoMol project (Tennyson et al. 2013) involves the use of two files. The largest file is the transitions file which has an entry for each line of the form I J AIJ, where AIJ is an Einstein A coefficient, and $\mathrm{I}$ and $\mathrm{J}$ are pointers to row number in the energy file. The energy file contains not only the energy of state but also rigorous quantum numbers, such as the total angular momentum and parity, state degeneracy and, optionally, approximate quantum numbers. Besides being efficient on storage, this format has the advantage that the energy levels can be updated after completion of the line list to either improve it using new data (Harris 
et al. 2006; Barber et al. 2014) or indeed to change the isotopologue under consideration (Harris et al. 2008).

A relatively compact data format does not alter the fact the basic line lists are huge and it is not practical to use them in their entirety in computational models of hot astronomical objects. One method of avoiding this is by importance sampling, as for example used in HITEMP (Rothman et al. 2010). However, given the importance of many weak transitions, this does not result in a drastic reduction in size of the line list. A second possibility is to use cross sections (Hill et al. 2013) and this option has been implemented on the ExoMol website (http://www.exomol.com/xsecs). Other possibilities, which are actively being pursued, including the use of k-coefficients and of opacity functions.

\section{Conclusions}

The task of forming opacity functions for the atmospheres of hot astronomical objects requires a very significant quantity of atomic and molecular data (Sharp \& Burrows 2007). The ExoMol project aims to provide the necessary information on the spectroscopy of hot molecules, with a particular emphasis on those molecules which are likely to be important for exoplanets. The present paper gives an overview of progress in this area.

\section{Acknowledgements}

This work is supported by ERC Advanced Investigator Project 267219. We thank the members of the ExoMol team for their collaboration on this project.

\section{References}

Allard, F., Hauschildt, P. H., Alexander, D. R., \& Starrfield, S. 1997, ARA $ध$ A, 35, 137

Azzam, A. A. A., Lodi, L., Yurchenko, S. N., \& Tennyson, J. 2013a, J. Chem. Phys., submitted Azzam, A. A. A., Yurchenko, S. N., \& Tennyson, J. 2013b, MNRAS, in preparation

Barber, R. J., Strange, J., Hill, C., et al. 2014, MNRAS, 437, 1828

Barber, R. J., Tennyson, J., Harris, G. J., \& Tolchenov, R. N. 2006, MNRAS, 368, 1087

Barton, E. J., Yurchenko, S. N., \& Tennyson, J. 2013, MNRAS, 434, 1469

Beaulieu, J. P., Tinetti, G., Kipping, D., et al. 2011, ApJ, 731, 16

Boyarkin, O. V., Koshelev, M. A., Aseev, O., et al. 2013, Chem. Phys. Lett., 568-569, 14

Campargue, A., Kassi, S., Pachucki, K., \& Komasa, J. 2012, Phys. Chem. Chem. Phys., 14, 802

Carter, S. \& Bowman, J. M. 1998, J. Chem. Phys., 108, 4397

Carter, S., Sharma, A. R., \& Bowman, J. M. 2012, J. Chem. Phys., 137, 154301

Coppola, C. M., Lodi, L., \& Tennyson, J. 2011, MNRAS, 415, 487

Dello Russo, N., Bonev, B. P., DiSanti, M. A., et al. 2005, ApJ, 621, 537

Dinelli, B. M., Miller, S., Achilleos, N., et al. 1997, Icarus, 126, 107

Engel, A. E., Doss, N., Harris, G. J., \& Tennyson, J. 2005, MNRAS, 357, 471

Geballe, T. R., Kulkarni, S. R., Woodward, C. E., \& Sloan, G. C. 1996, ApJ, 467, L101

Harris, G. J., Larner, F. C., Tennyson, J., et al. 2008, MNRAS, 390, 143

Harris, G. J., Polyansky, O. L., \& Tennyson, J. 2002, ApJ, 578, 657

Harris, G. J., Tennyson, J., Kaminsky, B. M., Pavlenko, Y. V., \& Jones, H. R. A. 2006, MNRAS, 367,400

Hill, C., Yurchenko, S. N., \& Tennyson, J. 2013, Icarus, (in press)

Jørgensen, U. G., Almlöf, J., \& Siegbahn, P. E. M. 1989, ApJ, 343, 554

Kozin, I. N., Law, M. M., Tennyson, J., \& Hutson, J. M. 2004, Comput. Phys. Commun., 163, 117

Langhoff, S. 1997, ApJ, 481, 1007 
Le Roy, R. J. 2006, DPotFit 1.1 A Computer Program for Fitting Diatomic Molecule Spectral Data to Potential Energy Functions, University of Waterloo Chemical Physics Research Report CP-662R, http://leroy.uwaterloo.ca/programs/

Le Roy, R. J. 2007, LEVEL 8.0 A Computer Program for Solving the Radial Schrödinger Equation for Bound and Quasibound Levels, University of Waterloo Chemical Physics Research Report CP-663, http://leroy.uwaterloo.ca/programs/

Leyland, P. C., Yurchenko, S. N., \& Tennyson, J. 2013, MNRAS, in preparation Li, G., Gordon, I. E., Le Roy, R. J., et al. 2013, J. Quant. Spectrosc. Radiat. Transf., 121, 78 Lodi, L. \& Tennyson, J. 2010, J. Phys. B: At. Mol. Opt. Phys., 43, 133001

Lodi, L. \& Tennyson, J. 2012, J. Quant. Spectrosc. Radiat. Transf., 113, 850

Lodi, L., Tennyson, J., \& Polyansky, O. L. 2011, J. Chem. Phys., 135, 034113

Lynas-Gray, A. E., Miller, S., \& Tennyson, J. 1995, J. Mol. Spectrosc., 169, 458

Mellau, G. C. 2011, J. Mol. Spectrosc., 269, 77

Moudens, A., Georges, R., \& Benidar, A., et al. 2011, J. Quant. Spectrosc. Radiat. Transf., 112, 540

Neale, L., Miller, S., \& Tennyson, J. 1996, ApJ, 464, 516

Nikitin, A. V., Rey, M., \& Tyuterev, V. G. 2011, Chem. Phys. Lett., 501, 179

Nikitin, A. V., Rey, M., \& Tyuterev, V. G. 2013, Chem. Phys. Lett., 565, 5

Patrascu, A. T., Yurchenko, S. N., \& Tennyson, J. 2013, MNRAS, in preparation

Pavanello, M., Adamowicz, L., Alijah, A., et al. 2012, Phys. Rev. Lett., 108, 023002

Pavlyuchko, A. I., Vasilyev, E. V., \& Gribov, L. A. 2012, J. Appl. Spectrosc., 78, 782

Polyansky, O. L., Kozin, I. N., Maĺyszek, P., et al. 2013a, J. Phys. Chem. A

Polyansky, O. L., Ovsyannikov, R. I., Kyuberis, A. A., et al. 2013b, J. Phys. Chem. A

Polyansky, O. L. \& Tennyson, J. 1999, J. Chem. Phys., 110, 5056

Polyansky, O. L., Zobov, N. F., Viti, S., et al. 1997a, ApJ, 489, L205

Polyansky, O. L., Zobov, N. F., Viti, S., et al. 1997b, Science, 277, 346

Rey, M., Nikitin, A., \& Tyuterev, V. G. 2013, Phys. Chem. Chem. Phys.,

Rothman, L. S., Gordon, I. E., Barber, R. J., et al. 2010, J. Quant. Spectrosc. Radiat. Transf., 111,2139

Schwenke, D. 1998, Faraday Discuss., 109, 321

Schwenke, D. W. 1996, J. Phys. Chem., 100, 2867

Schwenke, D. W. 2002, Spectra Chimica Acta A, 58, 849

Sharp, C. M. \& Burrows, A. 2007, ApJS, 168, 140

Sousa-Silva, C., Yurchenko, S. N., \& Tennyson, J. 2013, J. Mol. Spectrosc., 288, 28

Swain, M. R., Vasisht, G., \& Tinetti, G. 2008, Nature, 452, 329

Tashkun, S. A. \& Perevalov, V. I. 2011, J. Quant. Spectrosc. Radiat. Transf., 112, 1403

Tennyson, J. 2012, WIREs Comput. Mol. Sci., 2, 698, 10.1002/wcms.94

Tennyson, J., Harris, G. J., Barber, R. J., et al. 2007, Mol. Phys., 105, 701

Tennyson, J., Hill, C., \& Yurchenko, S. N. 2013, in , AIP Conference Proceedings (AIP, New York)

Tennyson, J., Kostin, M. A., Barletta, P., et al. 2004, Comput. Phys. Commun., 163, 85

Tennyson, J. \& Yurchenko, S. N. 2012, MNRAS, 425, 21

Tinetti, G., Vidal-Madjar, A., Liang, M.-C., et al. 2007, Nature, 448, 169

Underwood, D. S., Tennyson, J., \& Yurchenko, S. N. 2013, Phys. Chem. Chem. Phys., 15, 10118

Urru, A., Kozin, I. N., Mulas, G., Braams, B. J., \& Tennyson, J. 2010, Mol. Phys., 108, 1973

Voronin, B. A., Tennyson, J., Tolchenov, R. N., Lugovskoy, A. A., \& Yurchenko, S. N. 2010, MNRAS, 402, 492

Waldmann, I. P., Tinetti, G., Deroo, P., et al. 2013, ApJ, 766, 7

Wallace, L., Livingston, W., Hinkle, K., \& Bernath, P. 1996, ApJS, 106, 165

Wang, X.-G. \& Carrington, Jr., T. 2013, J. Chem. Phys., 138, 104106

Warmbier, R., Schneider, R., Sharma, A. R., et al. 2009, A\&A, 495, 655

Yadin, B., Vaness, T., Conti, P., et al. 2012, MNRAS, 425, 34

Yu, S., Miller, C. E., Drouin, B. J., \& Mueller, H. S. P. 2012, J. Chem. Phys., 137, 024304

Yurchenko, S. N., Barber, R. J., \& Tennyson, J. 2011a, MNRAS, 413, 1828 
Yurchenko, S. N., Barber, R. J., Tennyson, J., Thiel, W., \& Jensen, P. 2011b, J. Mol. Spectrosc., 268,123

Yurchenko, S. N., Barber, R. J., Yachmenev, A., et al. 2009, J. Phys. Chem. A, 113, 11845

Yurchenko, S. N., Tennyson, J., Barber, R. J., \& Thiel, W. 2013, J. Mol. Spectrosc.

Yurchenko, S. N., Thiel, W., \& Jensen, P. 2007, J. Mol. Spectrosc., 245, 126

Zobov, N. F., Polyansky, O. L., Le Sueur, C. R., \& Tennyson, J. 1996, Chem. Phys. Lett., 260, 381 